\title{
Indoor Localization Using Three dimensional Multi-PDs Receiver Based on RSS
}

\author{
Thesis by \\ Yinghao Liu \\ In Partial Fulfillment of the Requirements \\ For the Degree of \\ Masters of Science (Electrical Engineering)
}

King Abdullah University of Science and Technology

Thuwal, Kingdom of Saudi Arabia

Insert Date (Apr, 2018) 


\section{EXAMINATION COMMITTEE PAGE}

The thesis of Yinghao Liu is approved by the examination committee

Committee Chairperson: Mohamed-Slim Alouini

Committee Members: Boon S. Ooi, Tien Khee Ng, Kihong Park 
C)Apr, 2018

Yinghao Liu

All Rights Reserved 


\section{ABSTRACT \\ Indoor Localization Using Three dimensional Multi-PD Receiver Based on RSS \\ Yinghao Liu}

In modern life, there are many applications where positioning plays an important role. People have developed the global positioning system (GPS) to locate world wide position with error in decameter scales, which brings people much convenience. However, the accuracy of GPS is too low for indoor localization. The signals will drop down due to the signal attenuation caused by construction materials. With the welldeveloped GPS being indispensable for outdoor activities, many researchers have been also devoted to seeking an indoor positioning system to realize indoor localization with acceptable error. Indoor localization can be very useful in different situations, like locating, tracking, navigation and identification. For example, in the mall, locating the exact goods for customers can provide much convenience and benefits. Locating and tracking in the airport can greatly help passengers save their time and energy in reaching the destination. In another general scenario of identification, the population of observed targets is usually larger than just one. Hence, only with small error, indoor localization system (ILS) can be able to identify the targets despite the neighbors.

Due to the emerging and urging demands of increasing the accuracy of indoor localization, we propose a novel design of three dimensional (3-D). optical receiver for visible light communication (VLC) indoor positioning system. First, we model the optical wireless channel. Then we utilize modified triangulation method to obtain more robust receiver position by using at least two light-emitting diodes (LEDs) and one receiver consisting of nine photodetectors (PDs). Finally, the improved 
algorithm is implemented and the results are shown under our three dimensional multiple photodetectors (multi-PDs) structure receiver. In the simulation, we take the parameters of Lambertian radiation pattern, LEDs and PDs as those shown in [1].

To be noticed, our design of multi-PDs receiver is fully expanded into three dimensions compared with the pyramid receiver $(\mathrm{PR})$, which allows indoor positioning with our receiver structure to be more robust to the higher or corner positions. The details will be explained in the following sections. Based on Multiple-Photodiodebased Indoor Positioning algorithm [1], the indoor positioning algorithm is improved by redefining the optimization problem of obtaining the direction from receiver to LED and using weighted triangulation method to locate receiver position. We admit the solution under the redefined problem is not optimal to the actual problem. Yet, our given solution is better to that in [1] due to the existence of noise, which is reasonable and has been verified. 


\section{ACKNOWLEDGEMENTS}

First of all, I would like to express my gratitude to my MS/PhD advisor Professor Mohamed-Slim Alouini for his utmost support and advice over my research and life. He has been a great mentor and a helpful friend, always ready to inspire students. I admire him for both his endless knowledge and selfless mind.

Next, I would like to thank Kihong Park, research scientist at KAUST, for his patient and inspiring guide on my research progress and the expert advise on my research manuscripts.

I would also like to acknowledge Professor Boon S. Ooi, Senior Research Scientist Tien Khee Ng for considerate help in terms of perfecting the details.

I am indebted to KAUST for providing me the opportunity to study in a worldclass university with tremendously diverse and multi-cultural environment. I thank

all fellow members of communication theory lab for the simulating discussions and moral support.

I would like to express my profound gratitude to my friends and colleagues, who have accompanied and supported me all the time.

Finally, I sincerely thank my parents and my sister for their love and support. They are always there ready to motivate, encourage and help me in every possible way to pursue my dream. 


\section{TABLE OF CONTENTS}

\begin{tabular}{|l} 
Examination Committee Page \\
\hline
\end{tabular}

\begin{tabular}{ll} 
Copyright & 3 \\
\hline
\end{tabular}

\begin{tabular}{ll}
\hline Abstract & 4
\end{tabular}

\begin{tabular}{|l|l}
\hline Acknowledgements & 6
\end{tabular}

\begin{tabular}{ll}
\hline List of Abbreviations & 9
\end{tabular}

\begin{tabular}{ll}
\hline List of Figures & 10
\end{tabular}

\begin{tabular}{ll}
\hline List of Tables & 11
\end{tabular}

\begin{tabular}{lll}
\hline & Introduction & 12
\end{tabular}

1.1 Backgrounds and Review . . . . . . . . . . . . . . . . . . . . . . 12

1.2 Objectives and Contributions . . . . . . . . . . . . . . . 14

2 Positioning System 16

2.1 Transmitter . . . . . . . . . . . . . . . . . . . . . . . . 16

2.2 Channel Model . . . . . . . . . . . . . . . . . . . . . . . . . . . . . . 17

2.2 .1 Lambertian Radiation Pattern . . . . . . . . . . . . . . . . . . 17

2.2 .2 Lambertian Parameters . . . . . . . . . . . . . . . . . . . . . . 18

2.3 Receiver Design . . . . . . . . . . . . . . . . . . . . . . . . . . . . . . 19

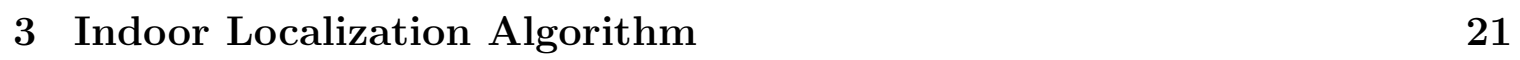

3.1 Pre-acknowledge . . . . . . . . . . . . . . . . . . 21

3.1.1 Coordinate Transformation . . . . . . . . . . . . . . . . . . . 22

3.1 .2 Triangulation . . . . . . . . . . . . . . . . . 23

3.2 Find the direction from receiver to transmitter . . . . . . . . . . . . . 25

3.2 .1 Ideal Scenario . . . . . . . . . . . . . . . . . . 26

3.2 .2 Non-Ideal Scenario . . . . . . . . . . . . . . . . . . 27

3.2 .3 Solution . . . . . . . . . . . . . . . . . . 28 
3.3 Find and Estimate Location . . . . . . . . . . . . . . . . . . . . . . . . 29

3.3 .1 Ideal Scenario . . . . . . . . . . . . . . . . . . . . . . . 30

3.3 .2 Non-Ideal Scenario . . . . . . . . . . . . . . . . . . . . 30

3.3 .3 Solution . . . . . . . . . . . . . . . . . . . . . 31

3.4 Localization Correction . . . . . . . . . . . . . . . . . . . . . . 32

3.4 .1 Cause of Error . . . . . . . . . . . . . . . . . . . . 32

3.4 .2 Solution . . . . . . . . . . . . . . . . . . . . . . . . 32

3.5 Proposed Improved Algorithm . . . . . . . . . . . . . . . . . . . 34

3.5 .1 Algorithm Without Localization Correction . . . . . . . . . 34

3.5 .2 Algorithm Without Localization Correction . . . . . . . . . . 34

$\begin{array}{lll}4 & \text { Simulation Results } & 37\end{array}$

4.1 Setup . . . . . . . . . . . . . . . . . . . . . . . . . . . . . . . 37

4.2 Results and Comparison . . . . . . . . . . . . . . . . . . 38

5 Concluding Remarks 44

5.1 Summary . . . . . . . . . . . . . . . . . . . . . 44

5.2 Future Application $\ldots \ldots \ldots \ldots \ldots$. . . . . . . . . . . . . 44

\begin{tabular}{ll}
\hline References & 46
\end{tabular}

\begin{tabular}{ll}
\hline Appendices & 47
\end{tabular} 


\section{LIST OF ABBREVIATIONS}

$\begin{array}{ll}3-D & \text { three dimensional } \\ \text { AOA } & \text { angle-of-arrival } \\ \text { FOV } & \text { field of view } \\ \text { GPS } & \text { global positioning system } \\ \text { HR } & \text { hemispheric receiver } \\ \text { ILS } & \text { indoor localization system } \\ \text { IR } & \text { infrared } \\ \text { LED } & \text { light-emitting diode } \\ \text { LEDs } & \text { light-emitting diodes } \\ \text { LOS } & \text { line of sight } \\ \text { multi-PDs } & \text { multiple photodetectors } \\ \text { PD } & \text { photodetector } \\ \text { PDs } & \text { photodetectors } \\ \text { PR } & \text { pyramid receiver } \\ \text { RF } & \text { radio frequency } \\ \text { RSS } & \text { received signal strength } \\ \text { TDMPIL } & \text { Three Dimensional Multi-PD Indoor Localiza- } \\ & \text { tion } \\ \text { TDR } & \text { three dimensional receiver } \\ \text { VLC } & \text { visible light communication } \\ \text { WCL } & \text { weighted centroid localization }\end{array}$




\section{LIST OF FIGURES}

2.1 Demonstration of the LOS channel. . . . . . . . . . . . . . . . . . . 18

2.2 (a) Top view of the receiver. (b) Front view of the receiver. . . . . . . 19

3.1 Coordinates transformation from (a) to (d). . . . . . . . . . . 24

3.2 Triangulation within two dimensions. . . . . . . . . . . . . . 25

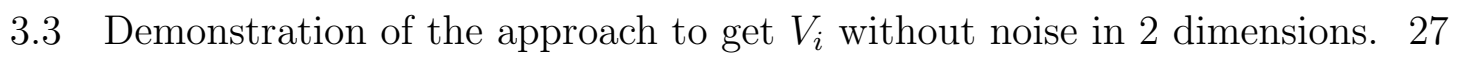

3.4 Demonstration of unreasonable error. . . . . . . . . . . . . . . 28

3.5 Localization correction. . . . . . . . . . . . . . . . . . . . . . 33

$4.1 \quad$ Comparison of the algorithms between with $W^{1}$ and without $W^{1}$ under our three dimensional receiver (TDR). . . . . . . . . . . . . . . . . 39

$4.2 \quad$ Comparison of the algorithms between with $W^{2}$ and without $W^{2}$ under

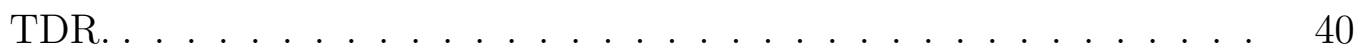

4.3 Comparison the positioning error of two different receiver structure when $\mathrm{z}=1.5 \mathrm{~m} . \ldots \ldots \ldots \ldots$. . . . . . . . . . . . . 41

4.4 Comparison the positioning error of two different receiver structure

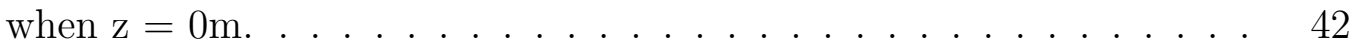

4.5 Comparison the positioning error of TDR LCT under different LCT

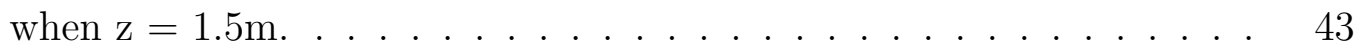




\section{LIST OF TABLES}




\section{Chapter 1}

\section{Introduction}

\subsection{Backgrounds and Review}

The GPS works out well with accuracy of several meters for the outdoor environments. However, the GPS signals can easily be blocked by the building walls, and we need to find another reliable positioning approach for the indoor environments. Indoor localization is the process to obtain the position of the target or device in the indoor environment. With precise positioning accuracy, there are a wide range of related applications and services, which include health sector, industry, disaster management, building management, surveillance and plenty of other sectors including smart buildings and smart cities [2].

There are many research projects studying indoor localization using Wi-Fi based, radio frequency $(\mathrm{RF})$ based, infrared (IR)-based and ultrasonic-based communication systems. Some systems conduct a Wi-Fi fingerprint localization method [3] using subsistent infrastructures, yet they need to build an environment-dependent database and can only get positioning accuracy with several meters. An indoor fingerprinting localization approach for Zig-Bee [4] is proposed to avoid collecting a large number of reference points in the tracking area by dividing the tracking area into subareas to achieve reasonable localization accuracy. The RADAR system in [5] implements a location service within several meters error utilizing the information obtained from an already existing RF data network to locate and track users inside buildings. But indoor environments often contain substantial amounts of metal and other such reflec- 
tive materials that affect the propagation of RF signals in non-trivial ways, causing severe multipath effects, dead-spots, noise, and interference, which would greatly effect the accuracy of positioning. Others like [6] can achieve good precision with wide coverage in positioning a mobile robot within an infrared sensor system, yet with the requirement of a very accurate design and device selection. In [7], an ultrasonic-based indoor positioning system for indoor environments with centimeter level error is presented but it can achieve high accuracy at the price of high cost for extra hardware.

The most popular RF-based indoor localization systems utilize existing wireless local networks to estimate position of the target, being able to provide better coverage than IR-based and ultrasonic-based positioning systems. The most common approaches being used in RF-, IR- and ultrasonic-based positioning systems are usually highly environment-dependent. The effects of multi-path and access points overlapping can greatly reduce the accuracy of indoor positioning.

VLC using visible light instead of RF signals for communication has drawn much attention in recent years. The light-emitting diode (LED) has been widely deployed because of long lifespan, high power efficiency, low deployment cost and illumination functions. Besides, LEDs can be used to modulate electric signals into optical signals without introducing interference even at high rates, which turns LEDs into communication devices with the potential of transmitting high speed data streams. Therefore, compared with RF-, IR- and ultrasonic-based positioning systems, positioning systems based on visible light communication have many merits such as no extra infrastructure, no electromagnetic interference and high accuracy.

Indoor localization based on received signal strength (RSS) of optical light has been studied in [8, 9, 10]. Centimeter-level errors can be achieved in [8] with the assumption of known height of the receiver. An array of PDs is mounted on the robot to obtain both RSS and angle-of-arrival (AOA) information in order to track the position of a moving robot in [9] with the assumption of known and fixed heights. 
Then another hybrid utilization of AOA and RSS information in VLC systems for 3-D localization is proposed in [10] and applies analytical learning rule to solve its non-linear and non-convex problems, which is pretty complex.

\subsection{Objectives and Contributions}

The main objective of the thesis is developing an improved algorithm of positioning the receiver by using the received signal strength.

The Miltiple-Photodiode-based Indoor Positioning algorithm [1] based on pyramid receiver is very original and effective, but there are some place to be improved from the perspective of the structure of the receiver and the actual environment.

This thesis focuses on a novel design of 3-D optical multi-PDs receiver and proposes an improved algorithm to enhance the performance of indoor localization based on [1]. In our indoor localization system, we assume that there are at least two LEDs on the ceiling with known positions in the room coordinate and then we use a 3-D multi-PDs receiver to locate its position based on RSS. The main contributions are as follows.

(a) A novel receiver structure is proposed, which gives better performance, especially when the height of the receiver is high, namely, $1.3 \mathrm{~m}-1.7 \mathrm{~m}$. The new structure contains nine PDs. Compared with pyramid structure, our multi-PDs receiver is fully expanded into three dimensions (more details about the distributions of the PDs are given in 2.3). This not only makes our receiver more sensitive to the optical signals coming from LEDs with much longer distance to the receiver but also but also enhances the coverage of the receiver.

(b) We show a critical problem neglected by the existing work when the noise is comparable to the optical signals received by PDs and then the solution is provided considering the practical environment. Intuitively, considering the 
white noise around all the PDs of the receiver, stronger optical signals coming from the near LEDs are more reliable than those from distant LEDs when the received signal strength is utilized to estimate the receiver's position. Hence, we should give more weights to the trustworthy optical signals rather than ignore the different effects coming from noise with the same distribution on the different received signals.

(c) The previous existing algorithm proposed in [1] is optimal under the circumstance of no noise. However, in reality, the performance of their algorithm highly depends on the actual position of the receiver or the optical signal strength. Generally speaking, we want the algorithm to be as robust as possible for indoor localization. Therefore, we revised the previous algorithm in terms of modifying triangulation with weights in order to obtain more accurate results. This can greatly lessen the error and has been verified by the simulation results. 


\section{Chapter 2}

\section{Positioning System}

In this chapter, we present the transmitter side information including the type, distribution and positions of LEDs in addition to the simulation environment. Then we describe the optical channel model from the prospective of LED radiation pattern, Lambertian pattern and the way to obtain Lambertain parameters. Finally, we give a detailed description of our 3-D receiver design.

\subsection{Transmitter}

The LEDs are taken as transmitters, and the optical light can be modulated into different signals with unique identification to be distinguished. Our indoor localization system assumes that there are at least two LEDs within the line of sight (LOS) link to the multi-PDs receiver, because only the direction (or the distance) from the receiver to the LED can be known if there is only one LED.

We model the wireless optical communication system in a room of size $5 \mathrm{~m} \times 5 \mathrm{~m} \times 3 \mathrm{~m}$. There are four LEDs uniformly distributed on the ceiling considered as transmitters.

Their positions are $\left(\frac{5}{3}, \frac{5}{3}, 3\right) \mathrm{m},\left(\frac{5}{3}, \frac{10}{3}, 3\right) \mathrm{m},\left(\frac{10}{3}, \frac{5}{3}, 3\right) \mathrm{m},\left(\frac{10}{3}, \frac{10}{3}, 3\right) \mathrm{m}$, respectively. The type of LEDs is Bridgelux LEDs (BXRA-56C5300-H-00), and the typical luminous flux of each LED is $50001 \mathrm{~m}$. 


\subsection{Channel Model}

The system takes irradiated LED optical signal at the LEDs and optical signal received by PDs as transmitted signal and received signal, respectively. The optical wireless channel is determined by the environment and LED irradiation pattern, which we assume as Lambertian emission.

\subsubsection{Lambertian Radiation Pattern}

Suppose $d_{j i}$ is the distance between the $j$-th photodetector (PD) $\{j: 1 \leq j \leq L\}$ and the $i$-th LED $\{i: 1 \leq i \leq K\}, \phi_{j i}$ is the irradiation angle of the optical signal from the $i$-th LED to the $j$-th PD with respect to the normal of the $i$-th LED (defined as the vector perpendicular to the LED plane), $\psi_{j i}$ is the incidence angle of the optical signal from the $i$-th LED to the $j$-th PD with respect to the normal of the $j$-th PD (defined as the vector perpendicular to the PD plane), and the effective area of PD is denoted as $A$. Then, the channel gain $H_{j i}$ from the $i$-th LED to the $j$-th PD through the LOS optical channel is

$$
H_{j i}=\frac{A(m+1)}{2 \pi d_{j i}^{2}} \cos ^{m}\left(\phi_{j i}\right) \cos ^{M}\left(\psi_{j i}\right) .
$$

Note that the Lambertian emission order $m$ is

$$
m=\frac{-\ln 2}{\ln \left(\cos \left(\phi_{1 / 2}\right)\right)},
$$

where $\phi_{1 / 2}$ is the LED semi-angle at half-power and

$$
M=\frac{-\ln 2}{\ln \left(\cos \left(\psi_{1 / 2}\right)\right)},
$$

where $\psi_{1 / 2}$ is the half power angle of incidence of a PD. The angles, $\phi_{1 / 2}$ and $\psi_{1 / 2}$, are determined by the properties of LEDs and PDs. Besides, channel gain $H_{j i}$ in (2.1) 
holds only if the optical LOS signal arrives within the field of view (FOV) of the PD. Otherwise, the PD detects no luminous power beyond its FOV.

Therefore, suppose the optical luminous flux $\Phi_{i}$ (in $\operatorname{lm}$ ) of the $i$-th LED is the transmitted luminous power, the received luminous power $P_{j i}$ transmitted from the $i$-th LED and received by the $j$-th PD is

$$
P_{j i}=\Phi_{i} H_{j i}=\Phi_{i} \frac{A(m+1)}{2 \pi d_{j i}^{2}} \cos ^{m}\left(\phi_{j i}\right) \cos ^{M}\left(\psi_{j i}\right)
$$

Here we give Fig. 2.1 to demonstrate the geometrical relationship of parameters in the LOS channel, where $O_{j}$ is the centrol point of the $j$-th PD and $N_{j}$ is the normal of the $j$-th PD.

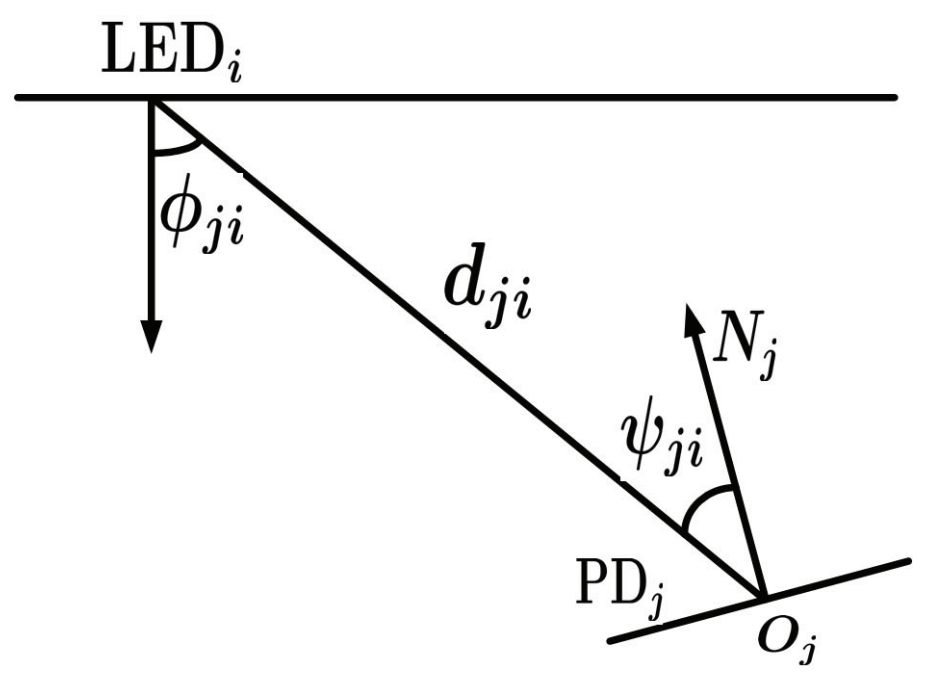

Figure 2.1: Demonstration of the LOS channel.

\subsubsection{Lambertian Parameters}

The definition of Lambertian parameters, $m$ and $M$, is given in 2.2.1. As mentioned above, the order of Lambertian emission $m=\frac{-\ln 2}{\ln \left(\cos \left(\phi_{1 / 2}\right)\right)}$ is determined by the property of the LED, $\phi_{1 / 2}$, which is the half-power angle of irradiance of the LED. Also in another word, the half-power angle is also the semi-angle of the LED at half power. 


\subsection{Receiver Design}

The existing typical angle diversity receivers can be separated into PR and hemispheric receiver $(\mathrm{HR})$ proposed in [11. Inspired by this, we propose a novel angle diversity receiver with nine PDs. There are three titled PDs uniformly distributed with the same elevation angle in the horizontal plane and six PDs vertically distributed, where two PDs are placed vertically (like $\mathrm{PD}_{4}$ and $\mathrm{PD}_{5}$ ) in Fig. 2.2(b) around each PD (like $\mathrm{PD}_{1}$ ) in Fig. 2.2(a). Note that there is another PD on the other side of $\mathrm{PD}_{4}$ and same for $\mathrm{PD}_{5}$.

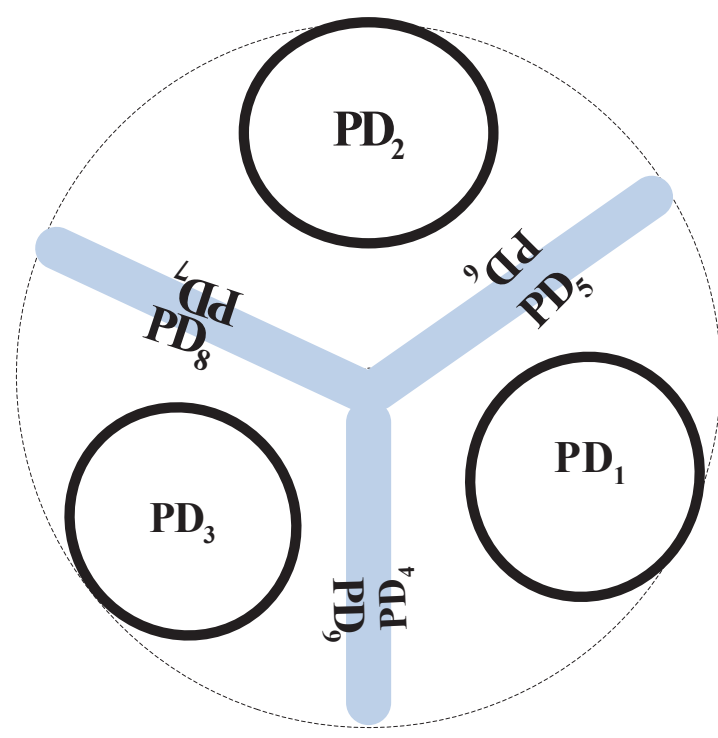

(a)

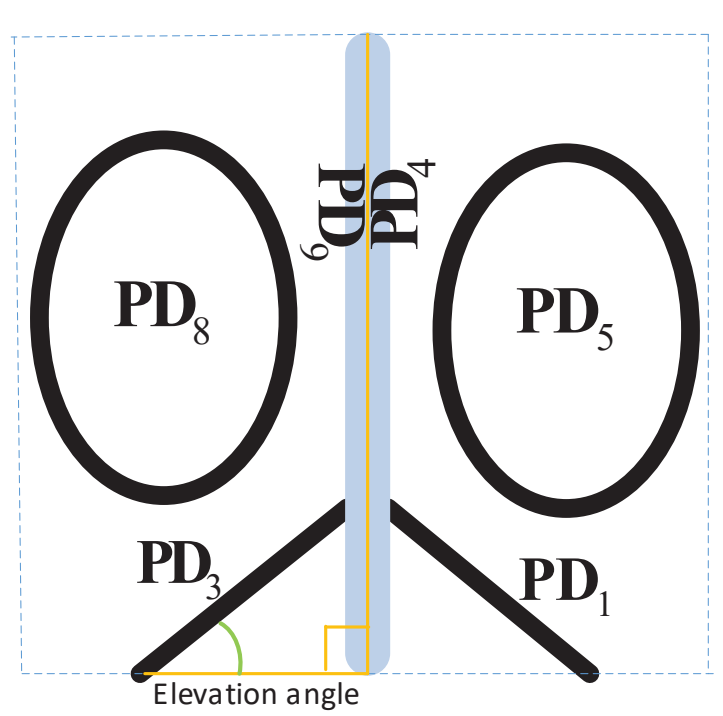

(b)

Figure 2.2: (a) Top view of the receiver. (b) Front view of the receiver.

Compared with $\mathrm{PR}$ and HR, our design is more robust to positions with higher height or around the corner in the room. For example, when the receiver is moved approximately with height from $0 \mathrm{~m}$ to $1.5 \mathrm{~m}$, the number of PDs in PR that can receive optical signals from a certain LED may decrease since the optical signals from distant LEDs may be out of FOV of some PDs. Yet, the number of PDs in our 3-D multi-PDs receiver being able to receive optical signal from a certain LED may increase due to the existence of vertically distributed PDs. Besides, the PDs in PR 
receive similar power since the PDs have similar orientation compared with our 3-D multi-PDs receiver. Intuitively, we can see that our three dimensional receiver will perform better considering the structure and application environment. The main reasons are

(1) It has more chance to receive optical signals from at least two or even more LEDs in the indoor positioning system.

(2) The more discrepancies the received signals from different LEDs hold, the less error the positioning system will produce. As for PR structures shown in [3], when the number of PDs is increased from three or four to nine, PR just gets more kinds of similar power from a greater number of LEDs, which cannot contribute much to positioning accuracy. 


\section{Chapter 3}

\section{Indoor Localization Algorithm}

In this chapter, we will introduce some pre-acknowledge of the indoor localization algorithm consisting of coordinate transformation and the weighted triangulation method, which helps us better understand the transformation of different coordinates and the following procedures within the positioning algorithm. Then, we describe the algorithm by decomposing it into three parts and explain each part in detail step by step. The ideal and non-ideal scenarios are both analyzed and the solutions are given based on the specific optimization problems. Besides, the systematic flaw is given with respect to the small distance between the central points of receiver and PD, and the localization correction is provided. At last, the algorithm tables are shown to summarize the detailed steps.

\subsection{Pre-acknowledge}

With the two parts of pre-acknowledge provided below, the approach to localize the position of receiver can be more straightforward. Since there are multiple PDs inside the receiver and the orientations of PDs are needed in the room coordinate, it is easy to obtain the orientations of PDs in the receiver coordinate and then convert these orientations into room coordinate, in which case the coordinate transformation is necessary. By collecting the optical signals received by the nine PDs, we try to obtain the directions from the receiver to LEDs in the room coordinate with the help of measurement of the accelerometer, after which the triangulation method is 
employed to calculate the position of the receiver in the room coordinate.

\subsubsection{Coordinate Transformation}

Through the accelerometer, the normal or orientation $N$ of the receiver can be obtained shown as in Fig. 3.1(a), which we assume is the $Z$-axis of the receiver coordinate. Now consider the coordinate $O_{a}$ is aligned with the room coordinate $O_{r}$, then coordinate $O_{a}$ can be known by shifting coordinate $O_{r}$ with a vector $v_{r \rightarrow a}$. The angle between $N$ and $Z_{a}$-axis is $\theta_{N}$ and the angle between the projection of $N$ and $X_{a}$-axis is $\phi_{N}$. We can get $O_{b}$ by rotating $O_{a}$ through $\phi_{N}$ angle along $Z_{a}$-axis, and then get $O_{c}$ by rotating $O_{b}$ through $\theta_{N}$ angle along new $Y_{b}$-axis. For any point $Q_{a}$ in coordinate $O_{a}$, if coordinate $O_{a}$ is rotated through $\phi_{N}$ along $Z_{a}$-axis, $O_{b}$ coordinate can be acquired. Let $Q_{b}$ denote the corresponding position in $O_{b}$ coordinate of fixed $Q_{a}$ point. According to linear algebra, we know the relation among $Q_{a}, Q_{b}$ and rotation $\operatorname{matrix} \Omega_{z}\left(\phi_{N}\right)$

$$
Q_{a}=\Omega_{z}\left(\phi_{N}\right) Q_{b}=\left[\begin{array}{ccc}
\cos \left(\phi_{N}\right) & -\sin \left(\phi_{N}\right) & 0 \\
\sin \left(\phi_{N}\right) & \cos \left(\phi_{N}\right) & 0 \\
0 & 0 & 1
\end{array}\right] Q_{b}
$$

Then we know the position of $Q_{a}$ in room coordinate is

$$
\begin{aligned}
Q_{r} & =v_{r \rightarrow a}+Q_{a} \\
& =v_{r \rightarrow a}+\Omega_{z}\left(\phi_{N}\right) \Omega_{y}\left(\theta_{N}\right) Q_{c}
\end{aligned}
$$

where $Q_{c}$ comes from the corresponding point $Q_{d}$ in coordinate $O_{d}$. The point $Q_{d}$, central point of a $\mathrm{PD}$, is known in the receiver coordinate $O_{d}$ because the distribution of PD is defined by us. But we do not know the misalignment angle $v_{N}$ between $O_{d}$ and $O_{c}$, which can help us obtain the positions of any points that are known in $O_{d}$ and unknown in $O_{c}$ (or $O_{a}$ ) coordinate, in order to calculate positions of these points 
in room coordinate. Suppose the misalignment angle between $O_{d}$ and $O_{c}$ is $v_{N}$, then the receiver coordinate $O_{d}$ can be acquired by rotating through $v_{N}$ along Z-axis in $O_{c}$. Applying the same rule above, there is a known point $Q_{d}$ in $O_{d}$ receiver coordinate, the position $Q_{r}$ in room coordinate $O_{r}$ is

$$
Q_{r}=v_{r \rightarrow a}+\Omega_{z}\left(\phi_{N}\right) \Omega_{y}\left(\theta_{N}\right) \Omega_{z}\left(v_{N}\right) Q_{d} .
$$

The geometric relation is shown in Fig. 3.1. If we know the discrepancy vector $v_{r \rightarrow a}$ and the misalignment angle $v_{N}$, we can easily get the position of every PDs in the room coordinate since we know PDs positions $Q_{d}$ in receiver coordinate $O_{d}$. As for $v_{N}$, we let it traverse from 0 to $2 \pi$ to find the most appropriate value, which is explained in our algorithm in the following part. To sum it up, we can get the luminous power matrix $P$ consisting of $P_{j i}$ if and only if we know $v_{N}$ and position of receiver $\Delta Q_{a}$ in room coordinate. This is useful in terms of producing power matrix $P$, which can be used to estimate the receiver position in simulation after adding noise with a certain range of $S N R$.

\subsubsection{Triangulation}

There are generally two main approaches being used to study indoor localization, trilateration [12, 13] and triangulation [10, 14].

Generally speaking, trilateration is the process of determining location of the target by measurement of distances, using the geometry of circles, spheres or triangles. triangulation is the process of determining the position of the target by forming triangles to it from known points when the angle of arrival is measurable. For 3-D positioning, there are at least two directions and two reference points mandatory for 3-D positioning compared with four distance and four reference points required in trilateration. 


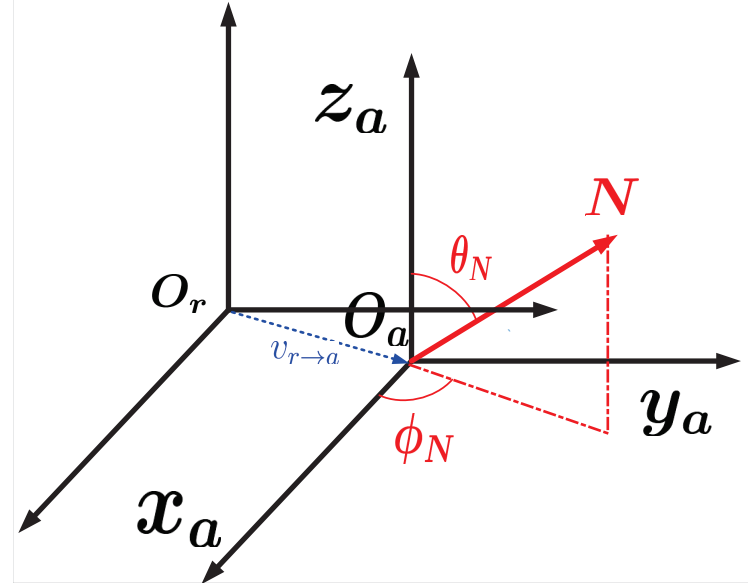

(a)

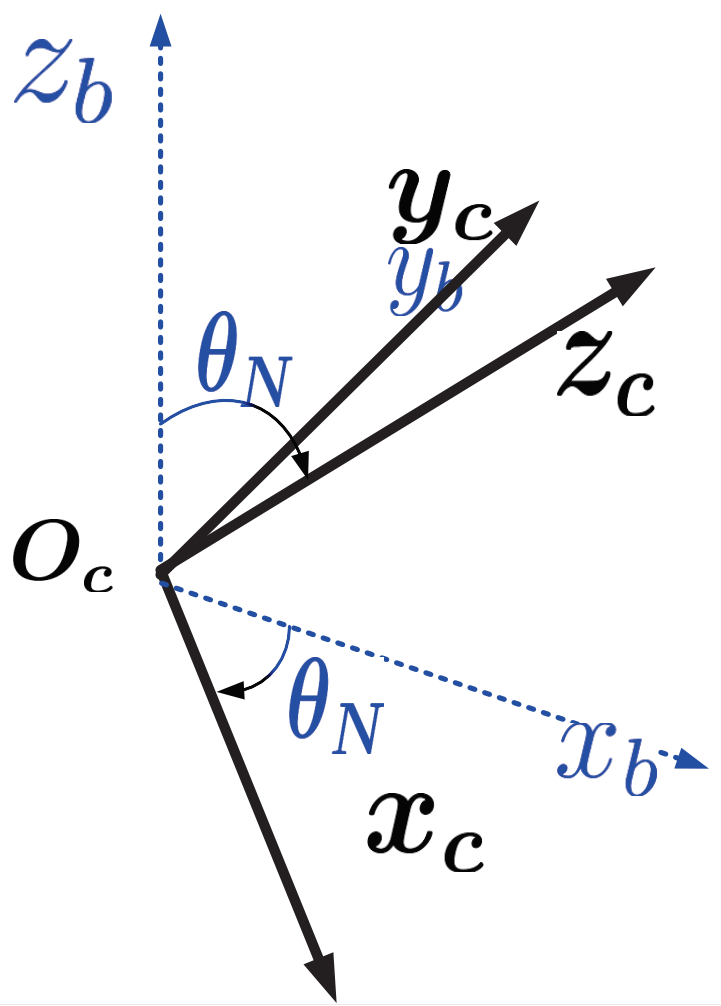

(c)

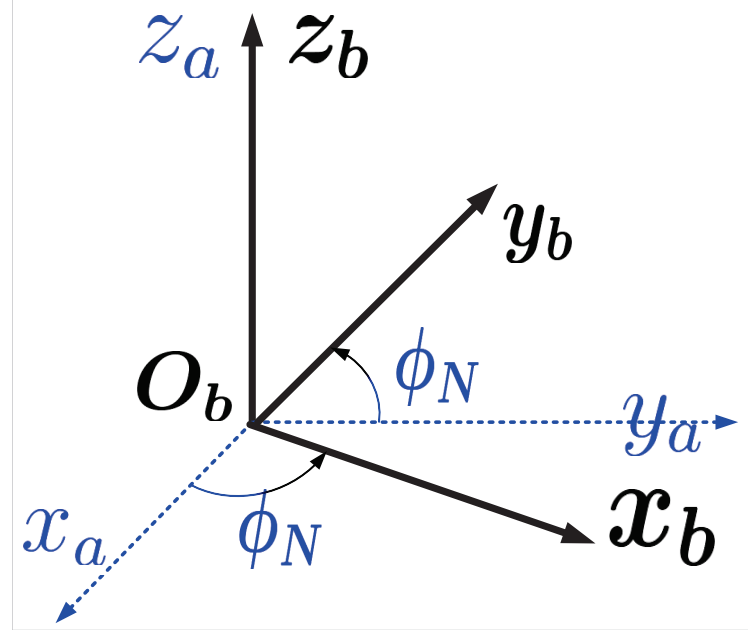

(b)

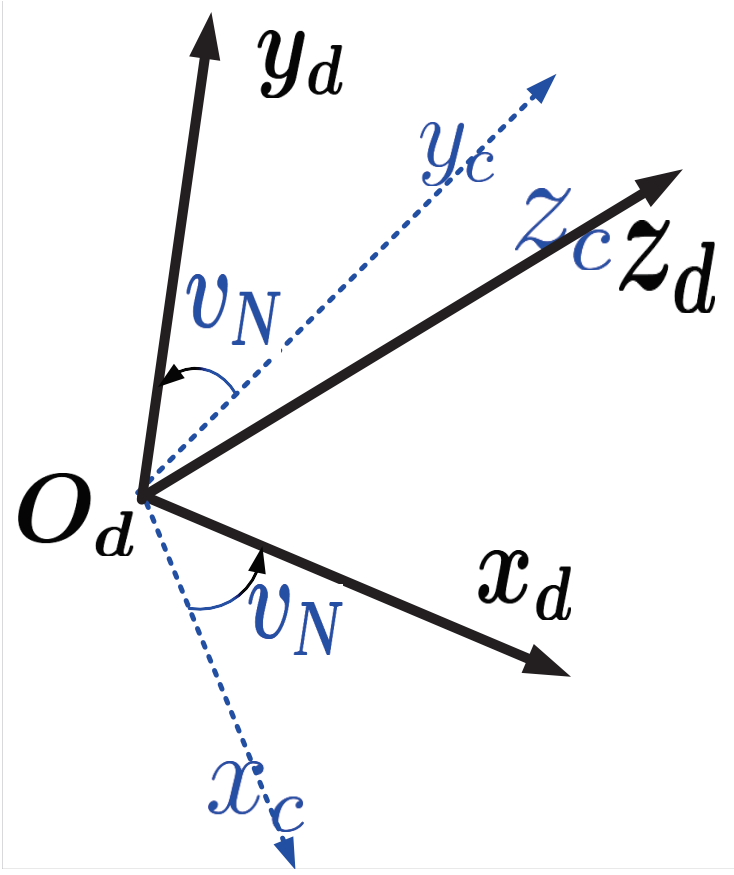

(d)

Figure 3.1: Coordinates transformation from (a) to (d). 
With triangulation, the location of the target can be determined by the intersection of several pairs of direction lines [15]. Take localizations in two dimensions for example shown in Fig. 3.2. $A$ and $B$ can be represented as the reference points, the known $\theta_{A}$ and $\theta_{B}$ can be converted into two lines as $L_{A T}$ and $L_{B T}$. Then the position of target $T$ can be calculated based on the predetermined coordinates of the reference points $A$ and $B$.

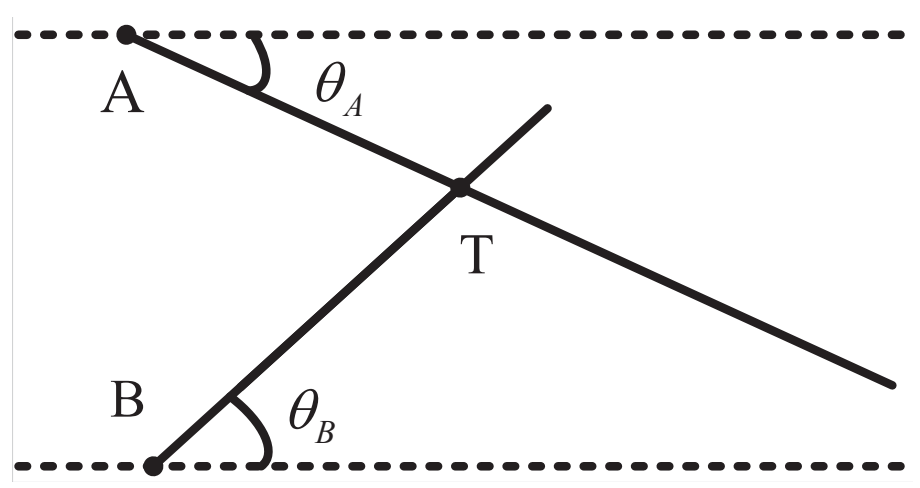

Figure 3.2: Triangulation within two dimensions.

\subsection{Find the direction from receiver to transmitter}

The pre-defined luminous power of received optical signal in 2.4 can form the received luminous power matrix $P$, where the $j$-th row represents the luminous power received by the $j$-th PDs from all LEDs and the $i$-th column represents the luminous power received by all PDs from the $i$-th LED. Through power matrix $P$, where the element $P_{j i}$ represents the optical signal luminous power received by the $j$-th $\mathrm{PD}$ from the $i$-th LED, we can calculate the estimated direction $\hat{V}_{i}$ from receiver towards the $i$-th LED.

Besides, in the aforementioned part 3.1, we try to divide the complex problem of obtaining the direction from receiver to LED in room coordinate into two parts of converting receiver coordinate into room coordinate with the assumed misalignment angle and obtaining the direction from receiver to LED. To be noticed, the accelerom- 
eter is necessary yet not sufficient for the coordinate transformation. The orientation given by the accelerometer can be considered as $Z$-axis of the receiver coordinate, then there is still a misalignment angle $v_{N}$ after we compensate for another two angles provided by the accelerometer between the room coordinate and semi-converted receiver coordinate. To solve this problem, the misalignment angle $v_{N}$ is assumed to be known, then we take the objective function, from which the position of receiver is estimated, as the evaluation function to estimate the actual misalignment angle corresponding with the minimum value of evaluation function. The details are provided in 3.3 .

\subsubsection{Ideal Scenario}

In (2.4), assuming that all LEDs have the same optical luminous power $\Phi$, we can let $\phi_{j i}=\phi_{i}$ and $d_{j i}=d_{i}$ for $\forall j \in\{1,2, \ldots, L\}$ since the small distance $\Delta_{j}$, between the center of the receiver and the $j$-th $\mathrm{PD}$, is negligible with respect to the distance between the $i$-th LED and the receiver center, $d_{i}$. Therefore, considering the $i$-th LED for $i \in\{1,2, \ldots, K\}$ and the $j$-th PD for $j \in\{1,2, \ldots, L\}$, we have

$$
\begin{aligned}
P_{j i} & =\Phi \frac{A(m+1)}{2 \pi d_{j i}^{2}} \cos ^{m}\left(\phi_{j i}\right) \cos ^{M}\left(\psi_{j i}\right) \\
& \approx \frac{C}{d_{i}^{2}} \cos ^{m}\left(\phi_{i}\right) \cos ^{M}\left(\psi_{j i}\right),
\end{aligned}
$$

where $C$ is defined as $\Phi \frac{A(m+1)}{2 \pi}$ that is known and constant.

Considering just the $i$-th LED, we can see $\cos \left(\psi_{j i}\right) \propto P_{j i}^{\frac{1}{M}}$. Hence, in the ideal scenario without noise, the planes perpendicular to the normal vectors of the $j$-th PD with length of $P_{j i}^{\frac{1}{M}}$ will intersect at a line (two PDs within LOS of the $i$-th LED) or a point (three or more PDs within LOS of the $i$-th LED), and the vector $V_{i}$ from the receiver to its projection on the line or the intersection point is pointing to the $i$-th LED. The geometric figure is shown in Fig. 3.3. Note $N_{j}$ is a vector with a certain 


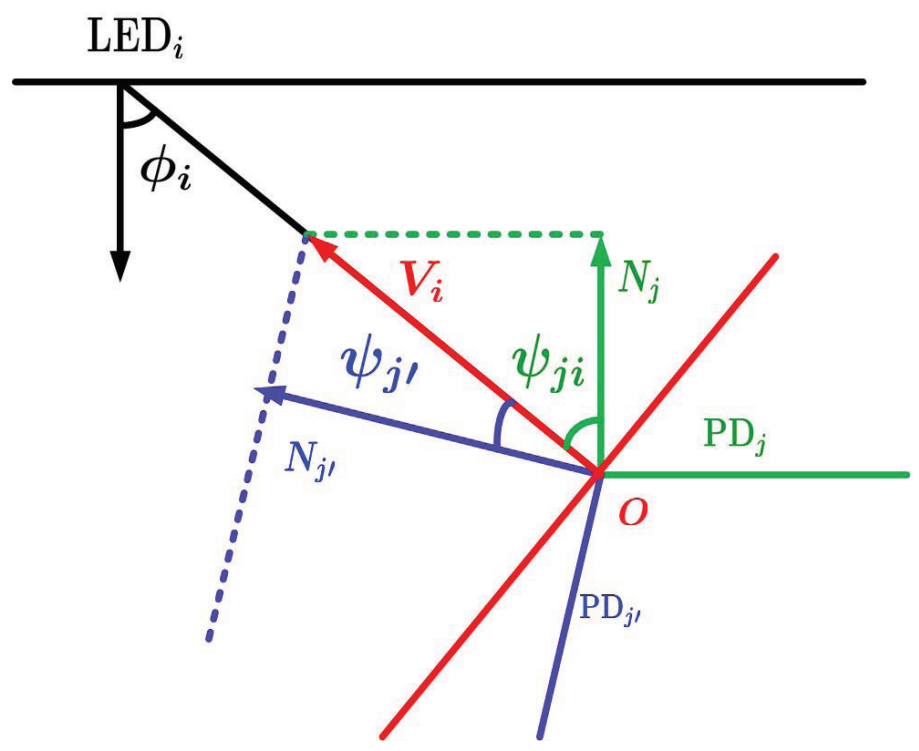

Figure 3.3: Demonstration of the approach to get $V_{i}$ without noise in 2 dimensions.

length for the rest context, rather than the orientation vector. The solutions provided in 1, 16] are optimal under the circumstance that there is no noise, which cannot be guaranteed in the real world.

\subsubsection{Non-Ideal Scenario}

In the non-ideal scenario or real environment, there is noise existing around each PD. Then the intersection line or point described in 3.2.1 may become a region. Therefore, we need to find a point $\omega$ which minimizes the summation of all the distances from $\omega$ to the projections of $\omega$ into the planes that are perpendicular to normals of PDs with

length $P_{j i}^{\frac{1}{M}}, N_{j}$. Lemma 1 in [16] gives a method to find the point $\omega$. However, they neglect a critical problem as shown in Fig 3.4, where the error would be unreasonable if $N_{j}$ is large and small $N_{j}$ ' is comparable to noise. Here $N_{j}$ ' is small with respect to $N_{j}$. Suppose $N_{j}$ ' is not big enough relative to noise $n_{j^{\prime}}$, then noise component on $N_{j}$ ' will lead $V_{i}$ far away from the correct direction, which could introduce huge error.

Therefore, $N_{j}$ with greater magnitude should be given more emphasis when we define the optimization problem, which helps obtain $\omega$ through minimizing summation 


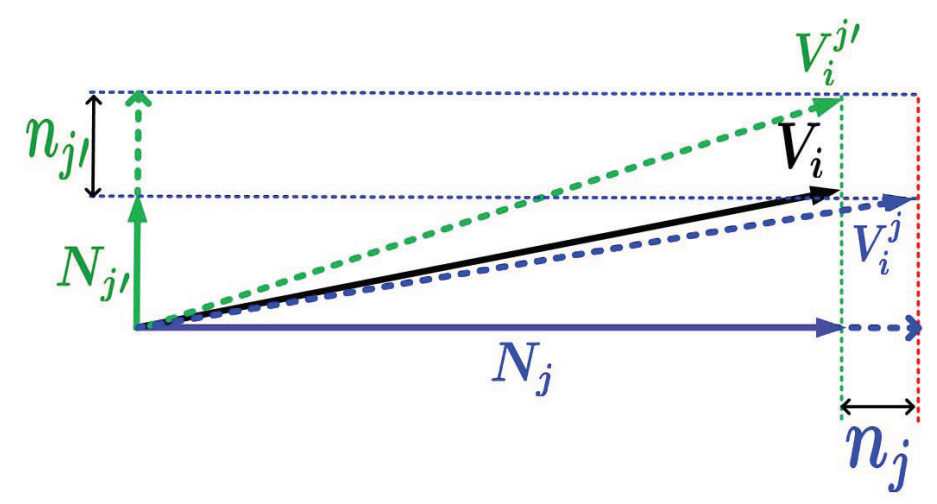

Figure 3.4: Demonstration of unreasonable error.

of all the distances from $\omega$ to the projection points $\omega_{j}$ on the $j$-th plane perpendicular to the $j$-th PD.

\subsubsection{Solution}

Suppose the misalignment angle between coordinate $O_{c}$ and coordinate $O_{d}$ is $v$, for the $i$-th LED, the orientation of PDs with length $P_{j i}^{\frac{1}{M}}$ are known, denoted as $N_{j}$. Then we have

$$
\begin{aligned}
& \omega_{j}-\omega=t N_{j}, \\
& \omega_{j}^{T} N_{j}=N_{j}^{T} N_{j} .
\end{aligned}
$$

Multiplying (3.6) by $N_{j}^{T}$ on the left side and substituting $N_{j}^{T} \omega_{j}$ with $N_{j}^{T} N_{j}$, we can get

$$
\begin{aligned}
t & =1-\frac{N_{j}^{T} \omega}{N_{j}^{T} N_{j}}, \\
\omega_{j}-\omega & =-\frac{N_{j} N_{j}^{T}}{N_{j}^{T} N_{j}} \omega+N_{j} .
\end{aligned}
$$


Let $E_{j}$ and $F_{j}$ denote $-\frac{N_{j} N_{j}^{T}}{N_{j}^{T} N_{j}}$ and $N_{j}$, we have

$$
\omega_{j}-\omega=E_{j} \omega+F_{j}
$$

Let $W_{j}^{1}=\left\|N_{j}\right\|^{2}$ denote weight put on the $j$-th plane since greater $N_{j}$ will introduce greater $W_{j}$. It means that we lay higher emphasis on the error resulting from $\left(\omega_{j}-\omega\right)$ part and the optimization problem to obtain $\omega$ or $V_{i}$ is defined as

$$
\begin{aligned}
\omega^{\star} & =\underset{\omega}{\operatorname{argmin}} f(\omega) \\
& =\underset{\omega}{\operatorname{argmin}} \sum_{j=1}^{L}\left(\left\|E_{j} \omega+F_{j}\right\|^{2} W_{j}^{1}\right),
\end{aligned}
$$

which gives us

$$
\omega^{\star}=-\left(\sum_{j=1}^{L} E_{j}^{T} E_{j} W_{j}^{1}\right)^{-1} \sum_{j=1}^{L} E_{j}^{T} F_{j} W_{j}^{1} .
$$

Denote $\sum_{j=1}^{L} E_{j}^{T} E_{j} W_{j}^{1}$ as matrix $A$ with rank $r$, we get $\omega^{\star}$ as following.

1. $r=1$ : This means there is just one $N_{j}$ that is none zero, hence $\omega^{\star}=N_{j}$.

2. $r=2$ : This problem is similar as $\omega=\min \|\omega\|^{2}$, subject to $A \omega=b$, where $b$ is $-\sum_{j=1}^{L} E_{j}^{T} F_{j} W_{j}^{1}$. The optimal solution is $\omega^{\star}=A^{T}\left(A A^{T}\right)^{-1} b$.

3. $r=3$ : We use matrix inverse operation in 3.12 .

Finally, the estimated direction from the receiver to the $i$-th LED is $V_{i}=\omega^{\star}$.

\subsection{Find and Estimate Location}

The directions, $V_{i}$ for $i \in\{1,2, \ldots, K\}$, from receiver position to LEDs can be estimated since the misalignment angle $v_{N}$ is assumed to be known as $v$. With the pre-known positions of LEDs as reference points mentioned in 3.1 .2 and these directions, we can use triangulation to estimate the position of the receiver in the room 
coordinate, which is of course under the assumption of the misalignment angle $v$.

\subsubsection{Ideal Scenario}

In the ideal scenario where there is no noise and no much small distance between the center of the receiver and the $\mathrm{PD}, V_{i}$ will be the exact direction pointing from the receiver to the $i$-th LED. According to the ideal directions $V_{i}$ and the positions of reference nodes or LEDs, the position of receiver, $s$, can be perfectly obtained as the intersection point of lines, where the $i$-th line is defined as

$$
r_{i}=h_{i}+t_{i} V_{i}
$$

where $\{i: 1 \leq i \leq K\}$ and $h_{i}$ denotes the position of the $i$-th LED. However, the directions from receiver to LEDs cannot be perfectly acquired, and this leads to the practical scenario and another optimization problem introduced in 3.3.2.

\subsubsection{Non-Ideal Scenario}

From the analysis above, it is known that in practical scenario these $K$ lines defined in 3.3.1 probably not intersect at one point due to the additive noise and the negligible small distance between the centers of receiver and PD. Therefore, we need to find the point $s$ that minimizes the summation of L2 norm squares of the vectors from $s$ to $s_{i}$, where $s_{i}$ is the point in line $L_{i}$ and most closest to point $s$. The fact that $s_{i}$ is within line $L_{i}$ tells us $\left(s_{i}-s\right) \perp V_{i}$, which can be written as $s_{i}^{T} V_{i}=s^{T} V_{i}$. From the expression of line 3.13 , we know

$$
\left(t_{i} V_{i}^{T}+h_{i}^{T}\right) V_{i}=s^{T} V_{i}
$$

Or equivalently,

$$
t_{i}=\frac{s^{T} V_{i}-h_{i}^{T} V_{i}}{V_{i}^{T} V_{i}}
$$


Substituting 3.15 into 3.14, we can get

$$
s_{i}-s=\left(\frac{V_{i} V_{i}^{T}}{V_{i}^{T} V_{i}}-I\right) s+\left(I-\frac{V_{i} V_{i}^{T}}{V_{i}^{T} V_{i}}\right) h_{i},
$$

where matrix $I$ represents appropriate identity matrix.

Previous work in [16] defines the objective function as the summation of all $L_{2}$ norms of the vectors from point $s$ to $s_{i}$ expressed in 3.16 and finds the optimal $s$ to minimize the objective function. However, we believe there still exists some space to improve the performance of localization by redefining the optimization problem.

\subsubsection{Solution}

Inspired by weighted centroid localization (WCL) in [17], here we define $W_{i}^{2}$ as $L_{2}$ norm of $V_{i}$. Greater $\left\|V_{i}\right\|^{2}$ indicates stronger received luminous power or smaller distance $d_{i}$ between the $i$-th LED and the receiver, where we regard error coming from smaller distance as important. Let $B_{i}=\frac{V_{i} V_{i}^{T}}{V_{i}^{T} V_{i}}-I$, the revised optimization problem can be transformed into

$$
\begin{aligned}
s^{\star} & =\underset{s}{\operatorname{argmin}} \sum_{i=1}^{K}\left(\left\|s_{i}-s\right\|^{2} W_{i}^{2}\right) \triangleq g(s) \\
& =\underset{s}{\operatorname{argmin}} \sum_{i=1}^{K}\left(\left\|B_{i} s-B_{i} h_{i}\right\|^{2} W_{i}^{2}\right) .
\end{aligned}
$$

Then we have the optimal $\hat{s}$ is:

$$
s^{\star}=\left(\sum_{i=1}^{K} B_{i}^{T} B_{i} W_{i}^{2}\right)^{-1} \sum_{i=1}^{K} B_{i}^{T} B_{i} h_{i} W_{i}^{2} .
$$

If matrix $\sum_{i=1}^{K} B_{i}^{T} B_{i} W_{i}^{2}$ is not full rank, we consider this problem as shown in the analysis of 3.12 .

We calculate $s^{\star}$ and the corresponding $g\left(s^{\star}\right)$ for each assumed $v$ changing from 0 
to $2 \pi$ and record the pair of values. Then the desired receiver position is estimated as $s^{\star}$ corresponding with the minimum $g\left(s^{\star}\right)$ over $v \in[0,2 \pi]$.

\subsection{Localization Correction}

We should have noticed the approximation from 3.4 to 3.5 holds with the prerequisite of ignoring the small distance between PD and the center of the receiver. Theoretically, the error coming from the small distance should be compensated, otherwise, it would be a limiting factor of this method to reduce the localization error in further study.

\subsubsection{Cause of Error}

Previously, we consider the receiver as an ideal point since the size of the receiver is usually small enough to be neglected compared with the distance between LED and receiver. Subsequently, the irradiation angles of the optical signals $\phi_{j i}$ from the $i$-th LED to the $j$-th PD are taken the same and denoted as $\phi_{i}$. However, strictly speaking, we know $\phi_{j i} \approx \phi_{i}$ rather than $\phi_{j i}=\phi_{i}$. Therefore, the error coming from the distance between PD and center of the receiver needs to be compensated.

\subsubsection{Solution}

The geometry figure of localization error and correction analysis is shown in Fig. 3.5 . Here $N_{j}$ is the normal of the $j$-th PD in room coordinate. $O_{j}$ represents the position of the $j$-th PD and $O$ denotes the original point of receiver coordinate. The actual $P_{j i}$ is the luminous power received by the $j$-th PD from the $i$-th LED, in which case the $j$-th PD is actually at point $O_{j}$. However, in our indoor localization procedures the small distance between $O_{j}$ and $O$ is neglected since it is relatively small compared with $d_{j i}$. Therefore, we need to calculate the power $P_{j i}^{h}$ as if the $j$-th PD is placed on 


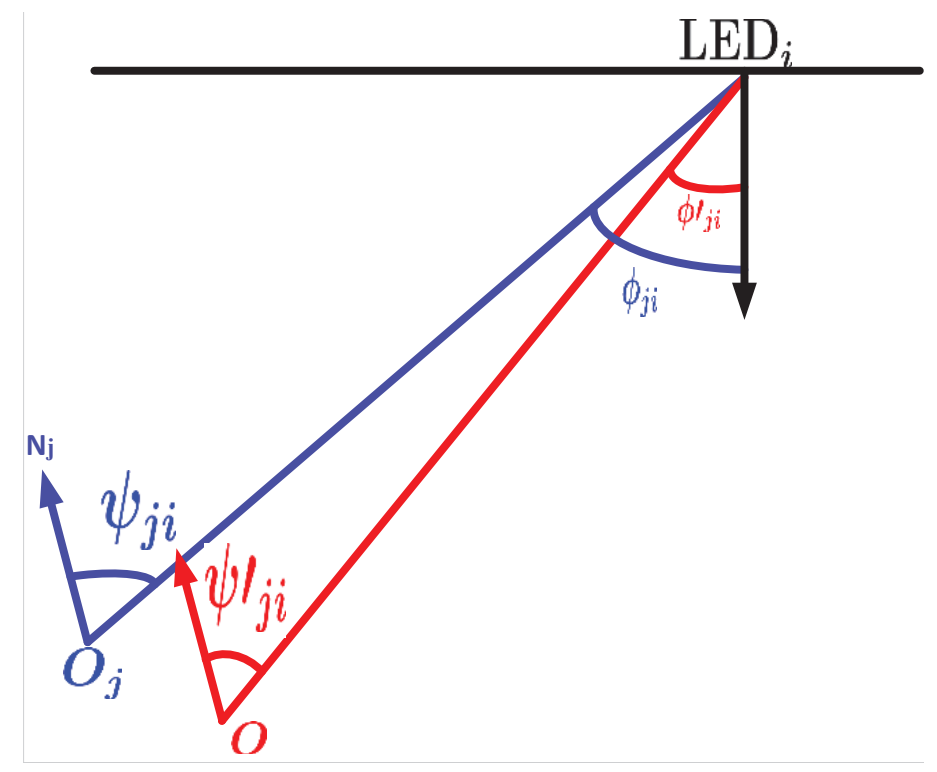

Figure 3.5: Localization correction.

$O$ point. From 3.4, we know

$$
P_{j i}=\frac{C}{\left\|h_{i}-O_{j}\right\|^{2}} \cos ^{m}\left(\phi_{j i}\right) \cos ^{M}\left(\psi_{j i}\right)
$$

where

$$
\cos \left(\phi_{j i}\right)=\frac{h_{i}(3)-O_{j}(3)}{\left\|h_{i}-O_{j}\right\|}
$$

and

$$
\cos \left(\psi_{j i}\right)=\frac{\left(h_{i}-O_{j}\right)^{T} N_{j}}{\left\|h_{i}-O_{j}\right\|\left\|N_{j}\right\|}
$$

Then we have

$$
P_{j i}=\frac{C\left(h_{i}(3)-O_{j}(3)\right)^{m}\left(\left(h_{i}-O_{j}\right)^{T} N_{j}\right)^{M}}{\left\|h_{i}-O_{j}\right\|^{2+m+M}} .
$$

However, the hypothesis luminous power received by the $j$-th $\mathrm{PD}$ at point $O$ is

$$
P_{j i}^{h}=\frac{C}{\left\|h_{i}-O\right\|^{2}} \cos ^{m}\left(\phi_{j i} \prime\right) \cos ^{M}\left(\psi_{j i} \prime\right)
$$


where

$$
\cos \left(\phi_{j i}{ }^{\prime}\right)=\frac{h_{i}(3)-O_{j}(3)}{\left\|h_{i}-O\right\|}
$$

and

$$
\cos \left(\psi_{j i} \prime\right)=\frac{\left(h_{i}-O\right)^{T} N_{j}}{\left\|h_{i}-O\right\|\left\|N_{j}\right\|}
$$

Then we have

$$
P_{j i}^{h}=\frac{C\left(h_{i}(3)-O(3)\right)^{m}\left(\left(h_{i}-O\right)^{T} N_{j}\right)^{M}}{\left\|h_{i}-O\right\|^{2+m+M}} .
$$

After the first time we calculate receiver position, we can obtain $O$ and $O_{j}$, then we can proceed localization correction and estimate the position of the receiver again by taking the hypothetical power matrix as the actual received one. Theoretically, or without noise, the procedure can be done as many times as we want, and the receiver position $s^{\star}$ should converge to the real position of receiver after proceeding localization correction enough times. However, the times of localization correction should be picked up carefully to try to avoid the estimated receiver position diverge from the real one due to the existence of optical noise.

\subsection{Proposed Improved Algorithm}

\subsubsection{Algorithm Without Localization Correction}

In this part, we introduce the algorithm to explain the indoor localization procedure, named Three Dimensional Multi-PD Indoor Localization (TDMPIL).

\subsubsection{Algorithm Without Localization Correction}

In this part, we introduce the algorithm table to explain the indoor localization procedure, named Three Dimensional Multi-PD Indoor Localization (TDMPIL) Through

the analysis in 3.4 .2 , we could enhance the indoor localization performance by iterating the TDMPIL algorithm. The improved version is denoted as the improved 


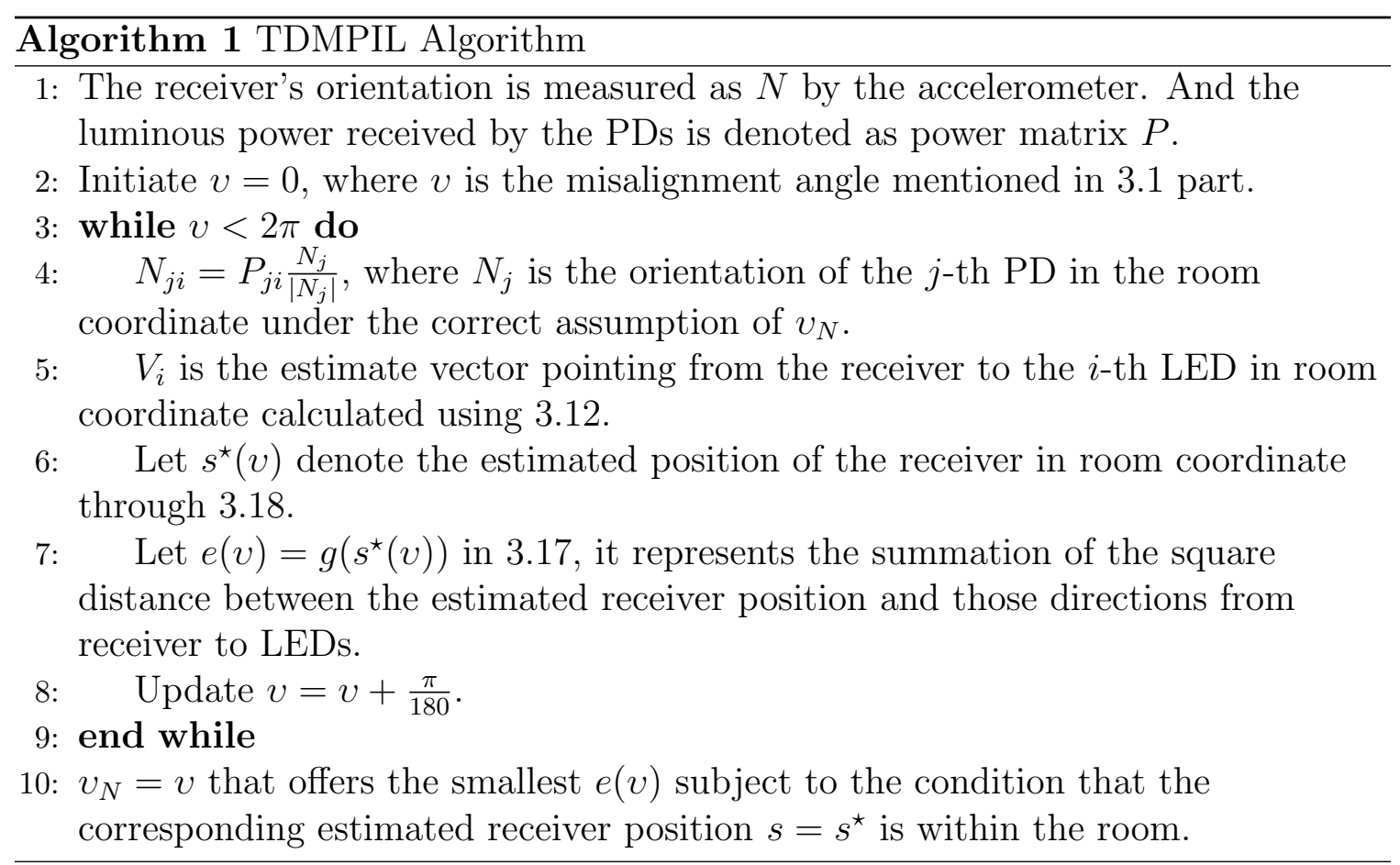

TDMPIL (ITDMPIL). 


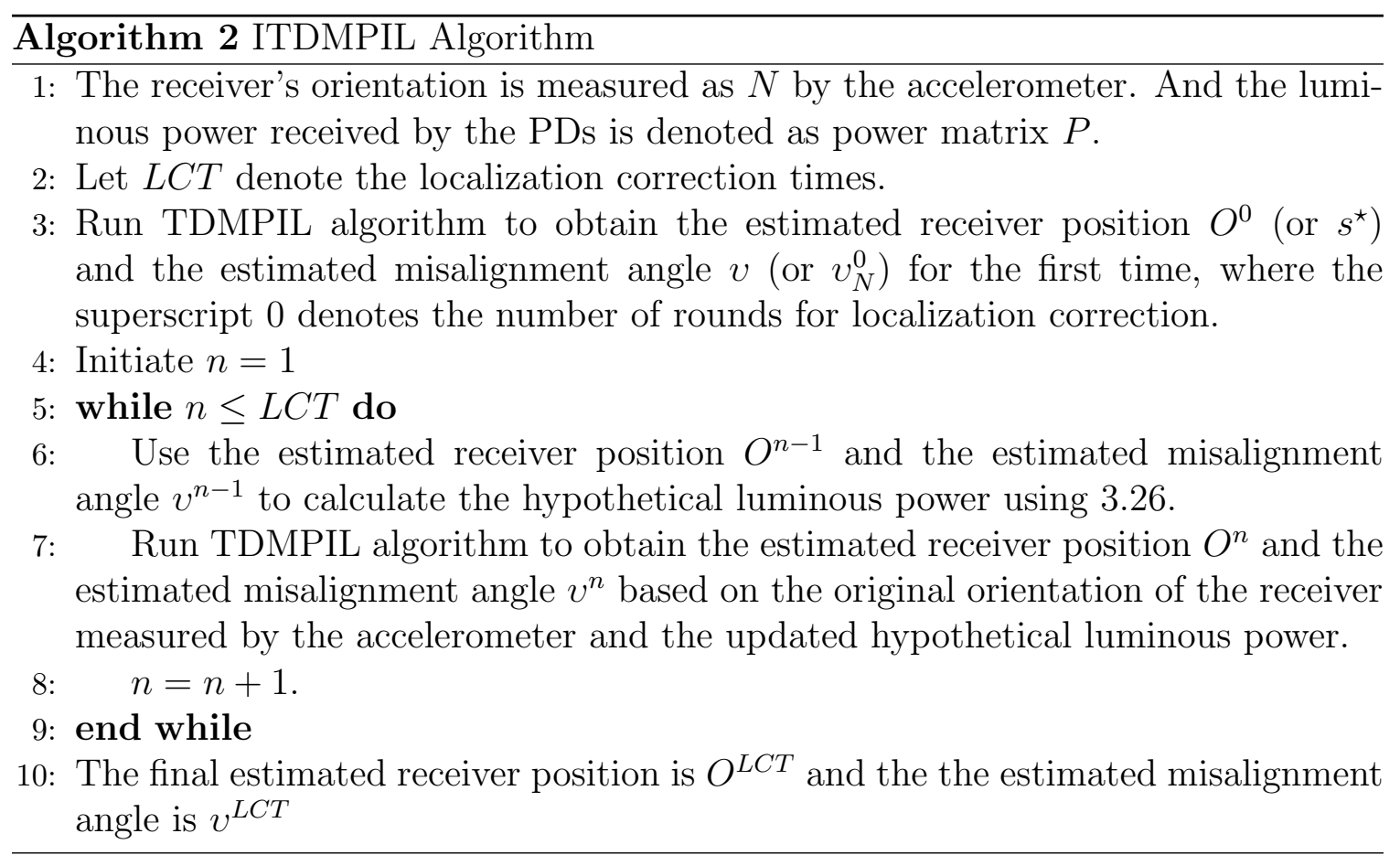




\section{Chapter 4}

\section{Simulation Results}

\subsection{Setup}

Before the results are given, the environment for the simulation should be notified. We model the wireless optical communication system in a room of size $5 \mathrm{~m} \times 5 \mathrm{~m} \times 3 \mathrm{~m}$. There are four LEDs uniformly distributed in the ceiling considered as transmitters,

that is, $\left(\frac{5}{3}, \frac{5}{3}, 3\right) \mathrm{m},\left(\frac{5}{3}, \frac{10}{3}, 3\right) \mathrm{m},\left(\frac{10}{3}, \frac{5}{3}, 3\right) \mathrm{m},\left(\frac{10}{3}, \frac{10}{3}, 3\right) \mathrm{m}$. The propagation of optical signal from LEDs is assumed to follow Lambertian pattern, and we take the Lambertian parameters $m$ and $M$ as 1 and 1.4, respectively, based on the experiments provided by [1]. At the receiver side, there are nine PDs spreading into three dimensions as our design, three of which are at the bottom and have the same elevation angle of $23^{\circ}$. The responsivity, effective area and FOV of each PD are $22 \mathrm{nA} \cdot \mathrm{lux}^{-1}, 15 \mathrm{~mm}^{2}$ and $1.22 \mathrm{rad}$.

Four typical points at two kinds of height are picked up to observe the positioning error under different noise, receiver structures and algorithms. In the simulation, we assume that the ambient noise is dominating the noise from the PD and accelerometer which are negligible in the simulation. And the noise variance $\sigma_{n}^{2}$ in lumens is estimated as $-180--120 \mathrm{~dB} \mathrm{~lm}^{2}$ when the luminous power of transmitted optical signal is $1000 \mathrm{~lm}$. Note that the error is averaged by 2000 times and the typical testing points $P 1, P 2, P 3, P 4$ and $P 5, P 6, P 7, P 8$ represent $(2.5,0,1.5) \mathrm{m},(2.5,2.5,1.5) \mathrm{m}$, $(1.25,1.25,1.5) \mathrm{m},(0,0,1.5) \mathrm{m}$ and $(2.5,0,0) \mathrm{m},(2.5,2.5,0) \mathrm{m},(1.25,1.25,0) \mathrm{m},(0,0,0) \mathrm{m}$, respectively. 


\subsection{Results and Comparison}

Under our three dimensional receiver structure, we pick up some typical points to demonstrate the effect of weight $W_{j}^{1}$ in the optimization problem (3.11). As shown in Fig. 4.1, reasonable weight $W_{j}^{1}$ can greatly reduce the error as a result of attenuating the disturbance of noise when we calculate the directions from the receiver towards LEDs. Therefore, we have verified the importance of weight $W_{j}^{1}$ in our redefined optimization problem (3.11). When the receiver position is at some typical points with height of $1.5 \mathrm{~m}$ in the room, our revised algorithm referring as $W_{j}^{1}$ performs better than that without $W^{1}$ and $W^{2}$. When $W^{1}$ exists but $W^{2}$ does not exist, the error magnitudes of corner points, $P 1$ and $P 4$, indicate that the neglected problem in previous work, as we mentioned in Fig. 3.4, can be solved with appropriate weights $W^{1}$. Here $W^{1}$ and $W^{2}$ denote the vectors composed of $W_{j}^{1}$ and $W_{i}^{2}$ for simplicity.

We want to know the influence of weight $W^{2}$, which works well especially when the receiver is near to one of the LEDs while far away from the others. Therefore, position $P 1$ is selected to show that $W^{2}$ can help make our improved algorithm more robust. $W^{2}$ can help rectify the mistake coming from the deflected directions calculated from the receiver to the LEDs due to noise, which is verified in Fig. 4.2. As for position like $P 2, P 3$ and $P 4$, it will not make much difference between triangulation and weighted triangulation.

Fig. 4.3 shows that our receiver structure is almost always better than PR when the height is $1.5 \mathrm{~m}$. The superiority is more obvious especially when the $S N R$ is high. To demonstrate this more clearly, Fig. 4.4 contains only two point $P 5$ and $P 7$ to illustrate that the errors coming from our receiver structure are also smaller than that from PR using the same improved algorithm at $0 \mathrm{~m}$ high level, which shows that our receiver is better than $\mathrm{PR}$. Note that the number of PDs in PR should also be nine when we compare these two different structures.

In Fig. 4.5, we can see that the positioning error when the number of localization 


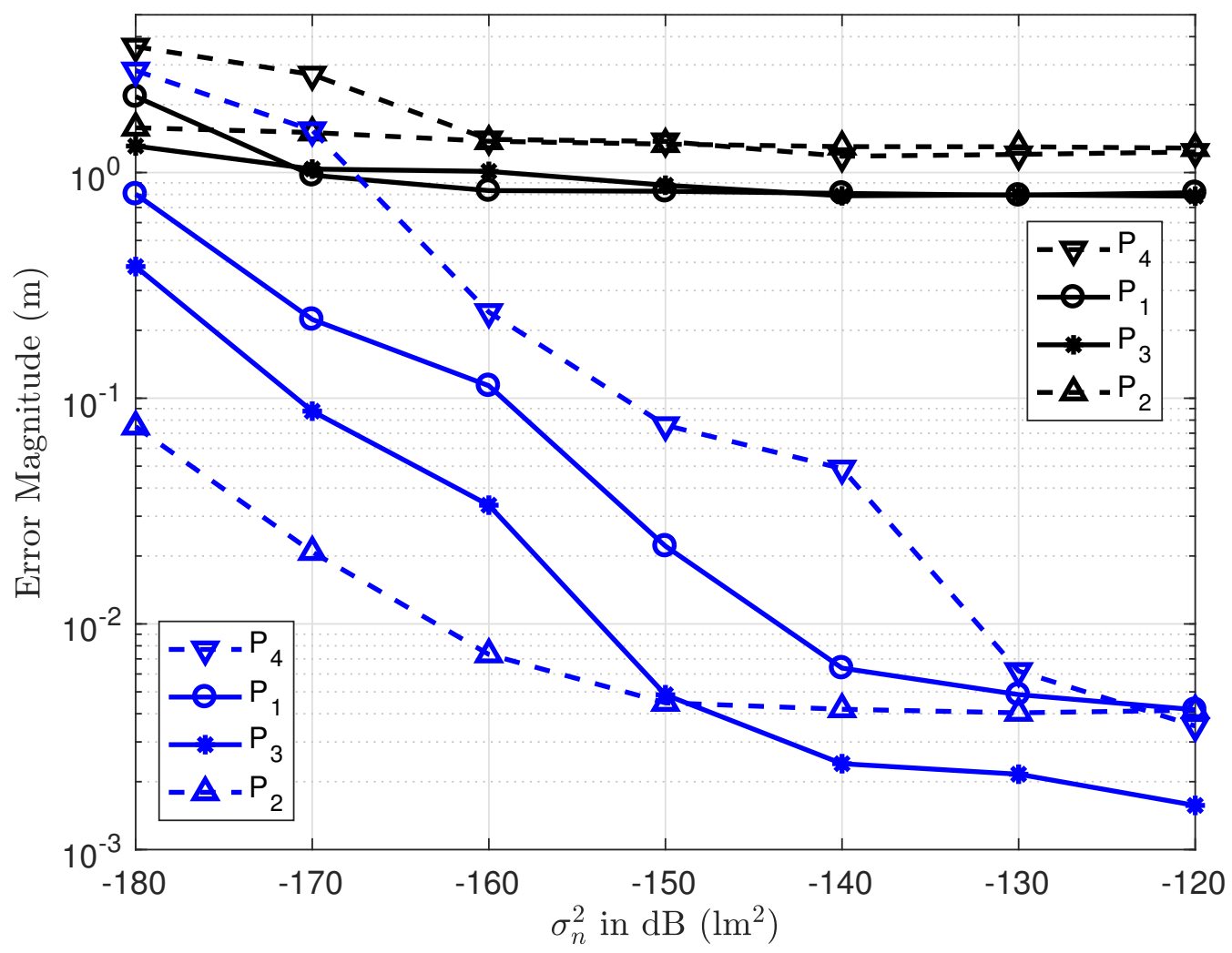

Figure 4.1: Comparison of the algorithms between with $W^{1}$ and without $W^{1}$ under our TDR. 


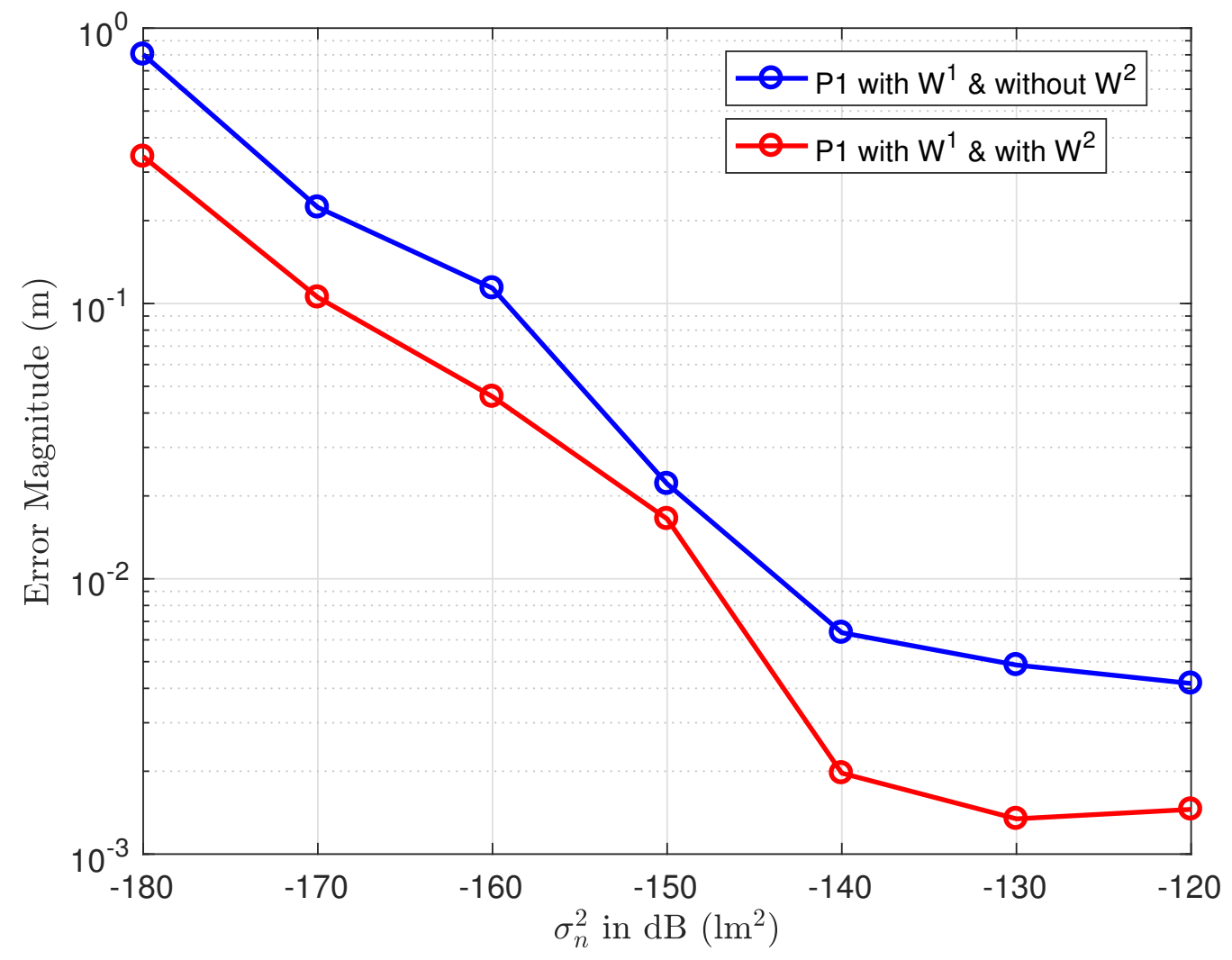

Figure 4.2: Comparison of the algorithms between with $W^{2}$ and without $W^{2}$ under TDR. 


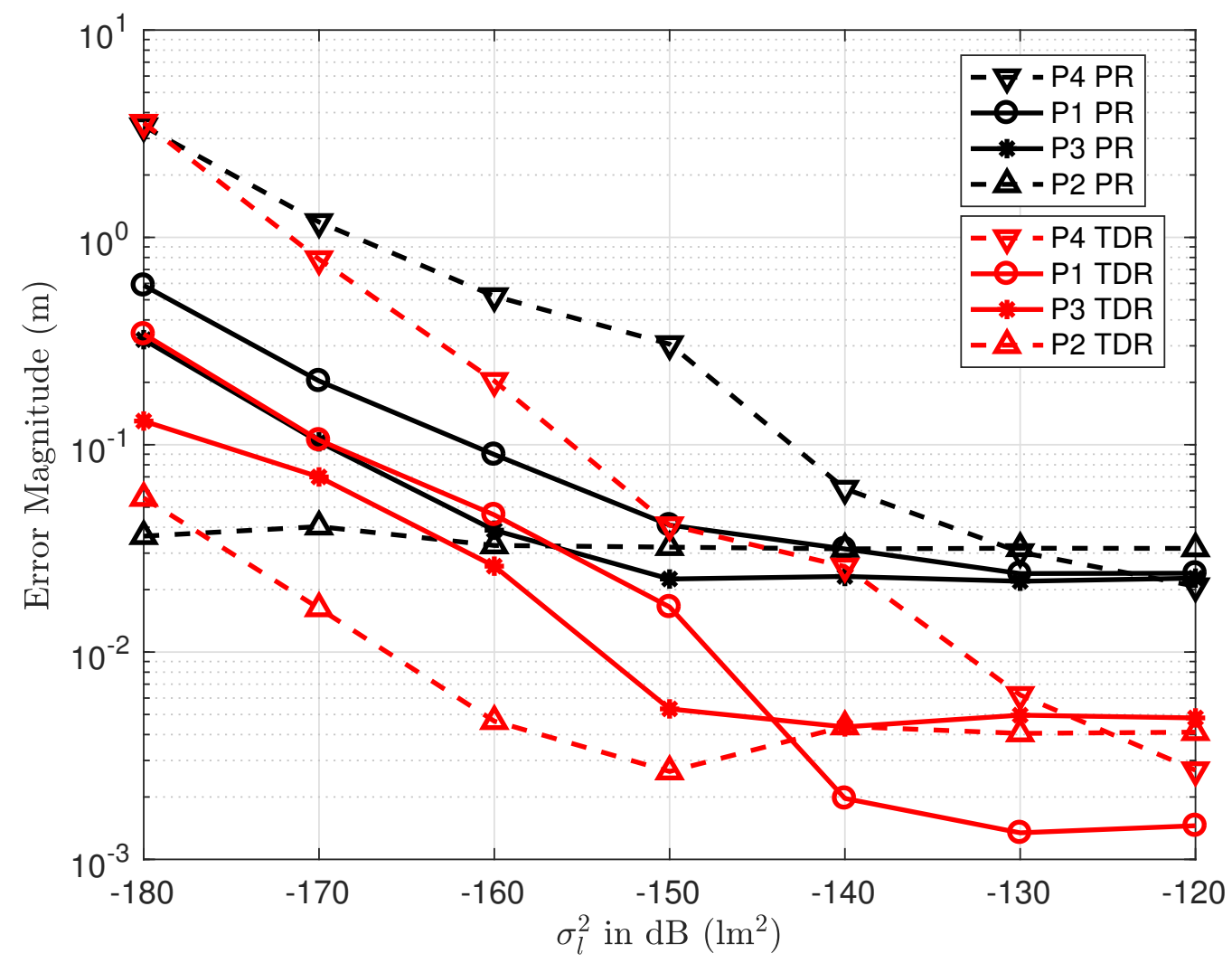

Figure 4.3: Comparison the positioning error of two different receiver structure when $\mathrm{z}=1.5 \mathrm{~m}$. 


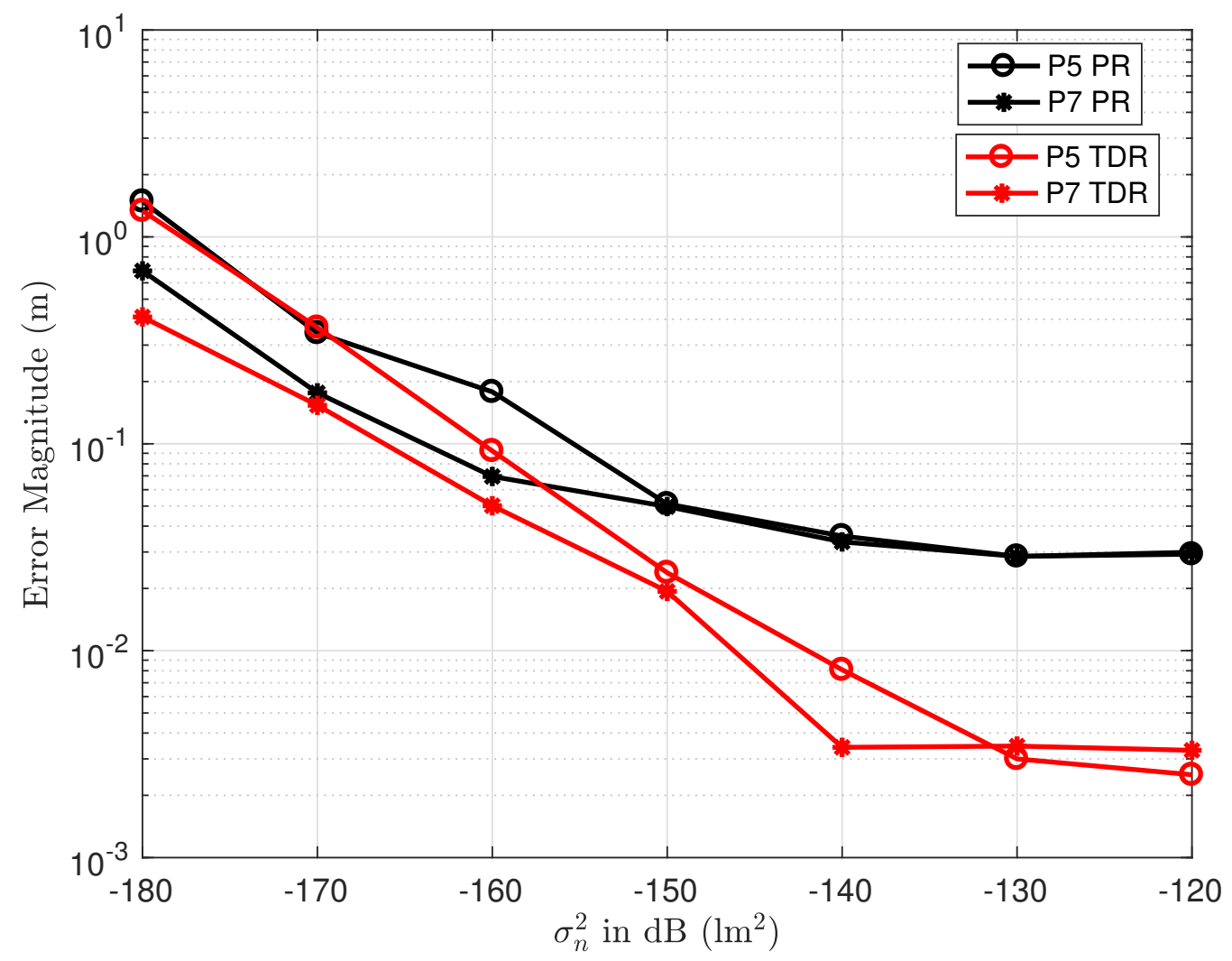

Figure 4.4: Comparison the positioning error of two different receiver structure when $\mathrm{z}=0 \mathrm{~m}$. 


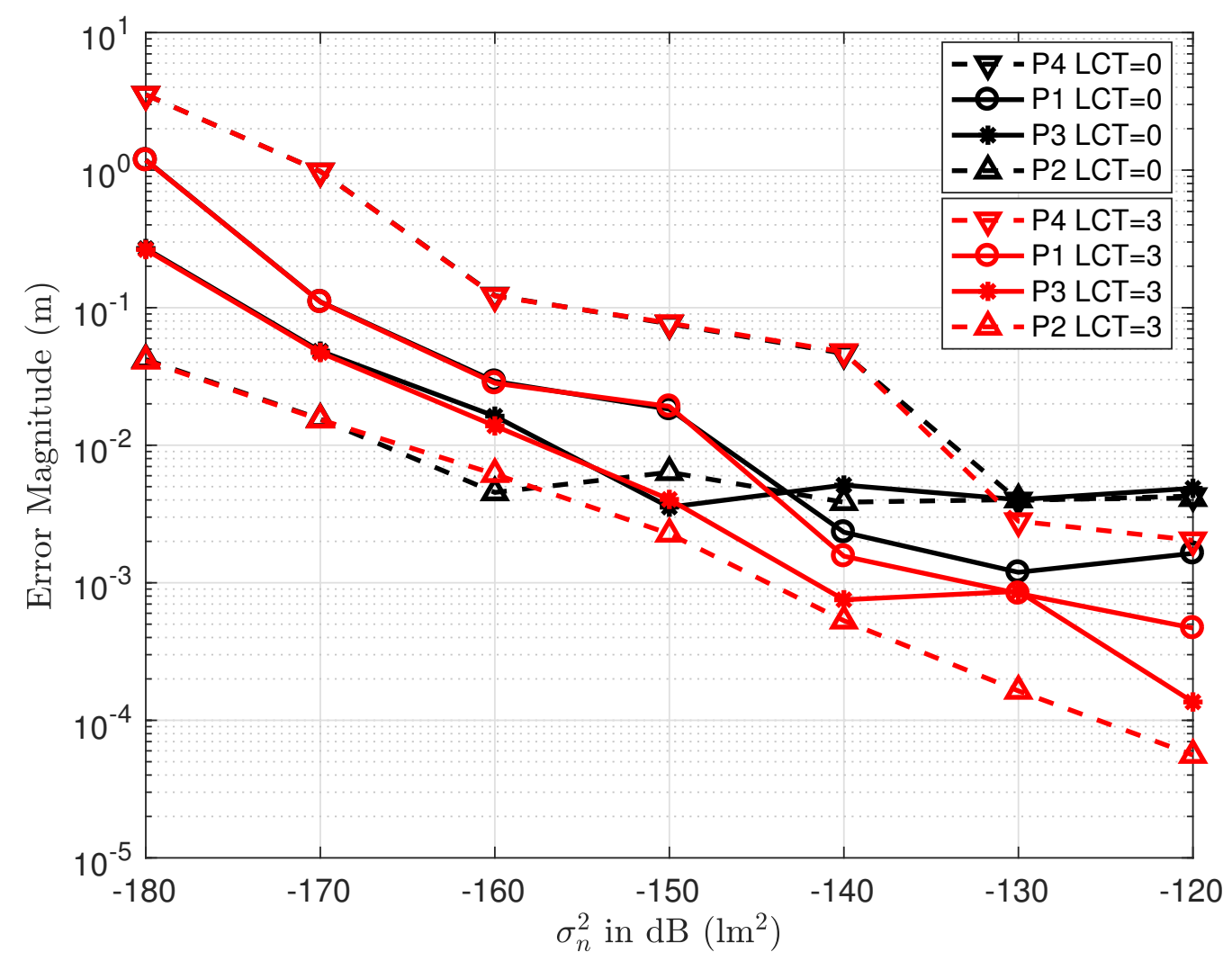

Figure 4.5: Comparison the positioning error of TDR LCT under different LCT when $\mathrm{z}=1.5 \mathrm{~m}$.

correction rounds is 3 is less than that without localization correction, which proves the effectiveness of localization correction. During the simulation, we also find that when there is no additive noise, once the correction is applied for just one time, the localization error will be fixed no matter how we increase the value of LCT, which is reasonable. 


\section{Chapter 5}

\section{Concluding Remarks}

\subsection{Summary}

We design a novel three dimensional structure receiver including nine PDs expanded into three dimensions. Using the accelerometer and the received optical signals detected by the PDs through coordinate transformation and the Lambertian equation, the receiver position is calculated by traversing the unknown misalignment angle $v$ from 0 to $2 \pi$. We propose the improved algorithm based on [1] with reasonable weights to reduce the positioning error, which can also solve the practical problem when the noise is comparable to some received signals within the FOV of PDs. The weighted triangulation can decrease the localization error when the receiver is placed at some edge positions. Simulation results reveal that our three dimensional receiver

performs better than PR angle diversity receiver. In addition, the comparison shows that our revised algorithm under three dimensional structure receiver is more robust to the noise.

\subsection{Future Application}

The applications for Indoor localization technology are immense. Take the shopping for instance, an app using ILS can offer an ideal route through the premises based on the customers own shopping list. Also, ILS can help track live sports results in real time, which can then be used in live broadcast and for live betting and gambling. In addition to commercial applications, let us assume the athlete is wearing a vest that 
can read bio-medical data, then the system is able to report like acceleration rate, heart and sweat rate, physical impact and numerous medical data in real time as well as exact location. Isn't it great?

Besides, ILS can help bolster workplace safety and security in warehouses by preventing accidents. With the help of ILS, Google will likely integrate this technology within their product suite which means some form of indoor advertising. Its too early to know exactly how this will all be connected but ILS is definitely a logical extension of the Google Maps product. The company may currently be using some newly acquired patents in the field of indoor wireless location technology to track assets in airports and other heavily trafficked locations.

It will be so exciting to see how these ILS technologies will go and help build enormous promising products to make the world a better place. 


\section{REFERENCES}

[1] M. Yasir, S.-W. Ho, and B. N. Vellambi, "Indoor position tracking using multiple optical receivers," J. Lightw. Technol., vol. 34, no. 4, pp. 1166-1176, Feb. 2016.

[2] F. Zafari, A. Gkelias, and K. Leung, "A survey of indoor localization systems and technologies," arXiv preprint arXiv:1709.01015, 2017.

[3] P. Jiang, Y. Zhang, W. Fu, H. Liu, and X. Su, "Indoor mobile localization based on wi-fi fingerprint's important access point," International Journal of Distributed Sensor Networks, vol. 11, no. 4, p. 429104, Jan. 2015.

[4] T. Alhmiedat, G. Samara, and A. O. A. Salem, "An indoor fingerprinting localization approach for ZigBee wireless sensor networks," European Journal of Scientific Research, vol. 105, no. 2, pp. 190-202, Jul. 2013.

[5] P. Bahl and V. N. Padmanabhan, "Radar: An in-building rf-based user location and tracking system," in INFOCOM 2000. Nineteenth Annual Joint Conference of the IEEE Computer and Communications Societies. Proceedings. IEEE, vol. 2. Ieee, 2000, pp. 775-784.

[6] E. Martin-Gorostiza, F. J. Meca-Meca, J. L. Lázaro-Galilea, D. Salido-Monzú, E. Martos-Naya, and A. Wieser, "Infrared local positioning system using phase differences," Nov. 2014, pp. 238-247.

[7] U. Yayan, H. Yucel, and A. Yazıcı, "A low cost ultrasonic based positioning system for the indoor navigation of mobile robots," Journal of Intelligent \& Robotic Systems, vol. 78, no. 3, pp. 541-552, Jun. 2015.

[8] W. Zhang and M. Kavehrad, "A 2-d indoor localization system based on visible light led," in Photonics Society Summer Topical Meeting Series, 2012 IEEE. IEEE, 2012, pp. 80-81.

[9] N.-T. Nguyen, N.-H. Nguyen, V.-H. Nguyen, K. Sripimanwat, and A. Suebsomran, "Improvement of the vlc localization method using the extended kalman filter," in TENCON 2014-2014 IEEE Region 10 Conference. IEEE, 2014, pp. 1-6. 
[10] A. Şahin, Y. S. Eroğlu, I. Güvenç, N. Pala, and M. Yüksel, "Hybrid 3-d localization for visible light communication systems," J. Lightw. Technol., vol. 33, no. 22, pp. 4589-4599, Nov. 2015.

[11] A. Nuwanpriya, S.-W. Ho, and C. S. Chen, "Indoor MIMO visible light communications: Novel angle diversity receivers for mobile users," J. Sel. Areas in Commun., vol. 33, no. 9, pp. 1780-1792, Sept. 2015.

[12] L. Li, P. Hu, C. Peng, G. Shen, and F. Zhao, "Epsilon: A visible light based positioning system," in Proc. NSDI, Apr. 2014, pp. 331-343.

[13] Z. Zhou, M. Kavehrad, and P. Deng, "Indoor positioning algorithm using lightemitting diode visible light communications."

[14] Y. S. Eroglu, I. Guvenc, N. Pala, and M. Yuksel, "AOA-based localization and tracking in multi-element VLC systems," in Proc. 2015 IEEE 16th Annual Wireless and Microwave Technology Conference (WAMICON), 2015, pp. 1-5.

[15] H. Liu, H. Darabi, P. Banerjee, and J. Liu, "Survey of wireless indoor positioning techniques and systems," IEEE Transactions on Systems, Man, and Cybernetics, Part C (Applications and Reviews), vol. 37, no. 6, pp. 1067-1080, 2007.

[16] M. Yasir, S.-W. Ho, and B. N. Vellambi, "Indoor positioning system using visible light and accelerometer," J. Lightw. Technol., vol. 32, no. 19, pp. 3306-3316, Oct. 2014.

[17] S. Subedi, G.-R. Kwon, S. Shin, S.-S. Hwang, and J.-Y. Pyun, "Beacon based indoor positioning system using weighted centroid localization approach," 2016, pp. 1016-1019. 


\section{A Papers Submitted and Under Preparation}

- Yinghao Liu, Kihong Park, and Mohamed-Slim Alouini, "' Indoor Localization Using Three dimensional Multi-PDs Receiver Based on RSS", Globecom. 